\title{
The expression of POMC and AgRP in brain and kidney tissues at different stages of diabetic nephropathy rats
}

Received 31 August 2020

Accepted 16 February 2021

\author{
Shasha Liu' ${ }^{1,2}$, Jingjing Da ${ }^{1,2,3}$, Jiayu Li ${ }^{1,2}$, Rong Dong ${ }^{1,2,3}$, Jing Yuan ${ }^{2,3}$, Yan Zha ${ }^{1,2,3, *}$ \\ ${ }^{1}$ Department of Biomedicine, Guizhou University School of Medicine, Guiyang, Guizhou Province, China \\ ${ }^{2}$ NHC Key Laboratory of Pulmonary Immunological Disease, Guizhou Provincial People's Hospital, Guiyang, Guizhou Province, China \\ ${ }^{3}$ Renal Division, Department of Medicine, Guizhou Provincial People's Hospital, Guiyang, Guizhou Province, China
}

Abstract

Objective: To explore the changes of proopiomelanocortin (POMC) and Agouti-Related Peptide (AgRP) expression in brain and kidney tissues under insulin intervention at different stages of diabetic nephropathy (DN) rats. Methods: The male Sprague-Dawley (SD) rats of DN were treated with high-fat diet for 8 weeks and induced by intraperitoneally injection of streptozotocin $(30 \mathrm{mg} / \mathrm{kg})$ for one time. Then DN rats were also injected insulin subcutaneously at $2-5 \mathrm{U} /(\mathrm{kg} \cdot 24 \mathrm{~h})$ from initiation of the streptozotocin. Kidney tissue, blood sample, and $24 \mathrm{~h}$-urine were collected to detect the ratio of kidney/body weight, blood glucose and 24-h urinary albumin excretion rate at different stages (4, 8, 12, and 16 weeks). Immunohistochemistry assay was used to measure the expression of POMC and AgRP at different stages of DN rats. Results: The DN rats were established successfully. With the progression of DN, blood glucose, 24-h urinary albumin excretion rate and kidney body weight ratio increased significantly, while decreased when insulin was injected. Immunohistochemistry showed that the expression levels of POMC were decreased gradually in brain and kidney tissues. Conversely, the expression of AgRP in kidney was highest at week 8 and then decreased gradually. The effect of insulin on normalizing POMC and AgRP expression in brain and renal tissues was also observed in DKD rats. Conclusion: With the progression of DN, the expression of POMC and AgRP in kidney tissues was observed at different stages of disease, and their expressions were significantly normalized by insulin. The mechanism of in situ expression of POMC and AGRP in kidney to the progression of DN needs further investigations.

Keywords

diabetic nephropathy $\bullet$ insulin $\bullet P O M C \cdot A g R P \cdot$ different disease stages

\section{Introduction}

Diabetes, a chronic disease, can lead to multiple complications. Diabetic nephropathy (DN), a major complication of diabetes, is the leading cause of end-stage renal disease [1]. In 2019, about a billion people worldwide will be living with diabetes, and that the number is expected to increase by $51 \%$ by 2045 . The prevalence of diabetes has increased significantly over the past decade [2]. Furthermore, diabtes and its complications have a significant impact on both the individual economy and the social economy. Global direct health expenditure on diabetes is expected to reach $\$ 845$ billion by 2045 , with China the world's second largest annual health expenditure on diabetes [3]. Although several therapy options such as sodium-dependent glucose transporters 2(SGLT-2) inhibitors are available for DN, many patients are still unable to stop the progression to end-stage kidney disease, and there is no definitive treatment for the disease[4]. Therefore, it has been a hot topic in the field of therapeutic study on the efficiency of DN.

Hypothalamus plays an important role in the maintenance of energy homeostasis in mammals and humans, which is the regulatory center of energy metabolism balance $[5,6]$. Peripheral organs that regulated by network of anorexigenic and orexigenic neuropeptides in the hypothalamus are involved in regulating body weight, feeding, and blood sugar and energy homeostasis [7]. Kidney is not only an organ for excreting metabolic waste but also an important metabolic organ contributed to energy homeostasis. However, the latter is often ignored. Besides the liver, glucose-6-phosphatase is only expressed in the renal cortex to perform the function of gluconeogenesis [8]. At the same time, the renal medullary consumes energy for transport of water, electrolyte and metabolic wastes, leading to zero net energy produced by the kidneys [9, 10]. Furthermore, few previous studies have paid attention to the metabolic function of the kidney.

Numerous studies have shown that proopiomelanocortin (POMC)-derived peptides and agouti-related peptide (AgRP) are dysregulated in central nervous system (CNS) in diabetes and obesity-related diseases [11]. The POMC neurons and AgRP neurons can well integrate peripheral and central inputs to generate central instructions for feeding behavior. It is reported that POMC neurons suppress appetite by releasing $\alpha$-melanocyte-stimulating hormone $(\alpha-M S H)$ that acts on anorexic melanocorticoid-4 receptors (MC4Rs) [12]. 
Conversely, AgRP and neuropeptide Y (NPY) were released from AGRP neurons, which promote appetite and increase energy intake. The abnormal release of hormone and neuropeptides will aggravate metabolic stress, leading to metabolic syndrome and diabetes. However, it remains poorly understood whether POMC and AgRP neuroendocrine activity is directly involved in kidney injury related with $D N$ progression. Therefore, male Sprague-Dawley (SD) rats were fed with high fat diet and injected low-dose STZ to establish a DN rat model, and we observed the relationship of POMC/ AgRP levels in hypothalamus and kidney tissue with blood sugar, urine protein, and the ratio of renal and body weight at different stages of disease and to explore the intervention effect of insulin on them. The current study was designed to provide a new strategy for the neuroendocrine regulation of "brain-kidney" axis on the pathogenesis of DN.

\section{Materials and Methods}

\subsection{Animals}

Male SD rats aged 6 weeks were provided by Chongqing Tengxin Biotechnology, which were fed in a controlled temperature $\left(21^{\circ} \mathrm{C} \pm 2{ }^{\circ} \mathrm{C}\right)$, standard humidity (55-65\%), and a $12 \mathrm{~h}$ light-dark cycle. Additionally, all animal experiments were approved by the Ethics Committee of Guizhou People's Hospital and the institutional animal care and welfare committee.

\subsection{Animals model establishment and group design}

All animals were adaptive to feeding a week which were randomly assigned to two groups: control (Con, $n=20$ ) and T2DN $(n=40)$. To establish insulin resistance model, the T2DN group were fed high-fat diet for 8 weeks, then the Con group was given normal feed. After 8 weeks, al the rats were fasted for $24 \mathrm{~h}$ and given free water to drink. Streptozotocin (30 mg/kg weight) was used to establish the T2DN model with a single intraperitoneal injection, and the Con group was intraperitoneally injected the same dose of solvent citric acid buffer $(0.01 \mathrm{~mol} / \mathrm{L}$ PBS, $\mathrm{pH}=4.5$ ). The tail vein blood glucose was continuously measured twice at $72 \mathrm{~h}$ after Stz injection. If the random blood glucose $\geq 16.7 \mathrm{mmol} / \mathrm{L}$, the T2DN model is established successfully. Next, the 40 rats that were successfully modeled were randomly assigned to two groups, namely, T2DN $(n=20)$ and insulin $(n=20)$. The rats in the insulin group were injected subcutaneously with 2-5 U/ (kg.24 h) insulin (Novo Nordisk, Denmark), after continuous administration. Three groups of rats were sacrificed with $4 \%$ chloral hydrate, $4,8,12$ and 16 weeks after successful modeling, respectively, and blood, urine, brain, and kidney specimens were collected. Blood samples were collected by abdominal aortic puncture at the time of death, and the serum was centrifuged and frozen at $-20^{\circ} \mathrm{C}$. Then kidney and brain tissues were excised and soaked in $4 \%$ of neutral formaldehyde or liquid nitrogen.

\subsection{Measurement of physiological and biochemical func- tion}

Blood was taken from the tail vein of rats, and a blood glucose test chip was used to measure blood glucose. The urine was collected and centrifuged $24 \mathrm{~h}$ before the rat samples were killed. A microliter of supernatant was dropped onto an ultra-precision spectrophotometer to determine the protein concentration. Each sample was measured three times.

\subsection{Histopathological analysis of kidney}

Before tissue collection, rats in each group were fasted for $12 \mathrm{~h}$ and then killed under chloral hydrous anesthesia. The kidney and brain tissues were harvested and then were fixed in 4\% paraformaldehyde overnight after paraffin embedding. And $2 \mu \mathrm{m}$-thick sections were stained with hematoxylin and eosin. From kidney and brain tissues, the random fields were captured at $40 \times$ magnification using a high-resolution video camera (Leica) connected to a light microscope (Leica DM 300 LED).

\subsection{Immunohistochemistry for POMC and AGRP expression in kidney and brain tissues}

Deparaffinization and hydration of $4 \mu \mathrm{m}$-thick renal and brain tissue sections, $3 \% \mathrm{H}_{2} \mathrm{O}_{2}$ is used to block endogenous peroxidases and high pressure was used for antigen retrieval. After incubation of primary antibodies POMC (Abcam, 1:100) and AgRP (Boster, 1:80) overnight at $4^{\circ} \mathrm{C}$, horseradish peroxidase-labeled goat anti-rabbit (Abcam, 1:200) or monkey antigoat polyclonal antibody (Abcam, 1:200) was added to incubate for $90 \mathrm{~min}$ at room temperature and $30 \mathrm{~min}$ in $37^{\circ} \mathrm{C}$. The sections were staining by DAB, counterstained with hematoxylin, dehydrated, cleared, and sealed sheet with neutral gum. Ten field areas were randomly selected under with an Upright Metallurgical Microscope. An automated image analysis system (Image-Pro Plus 6.0) was used for measuring the density of positive nerve fibers and the integrated optical density (IOD), and the values were compared with others.

\subsection{Statistical analysis}

The experimental data were presented as means \pm SD. Statistical analysis was displayed using GraphPad Prism 6.0. Independent-samples $t$ test was used to test the difference between two different population means. One-way ANOVA multiple comparison was used for data analysis with only one variable. $P<0.05$, the difference was statistically significant.

\section{Results}

\subsection{Changes of blood glucose and urinary albumin excretion rate in different stages of DN rats}

To determine whether the T2DN model was successfully established and observe the changes of blood glucose levels in different periods, the tail venous blood of rats was taken to examine 
A

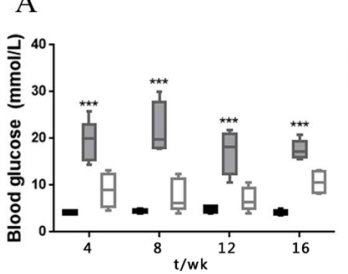

$\mathrm{D}$
B

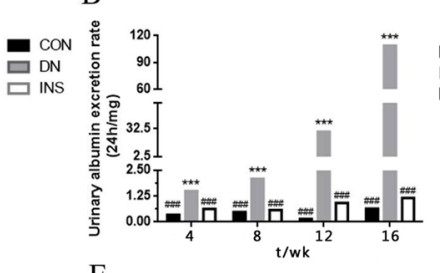

$\mathrm{E}$
C

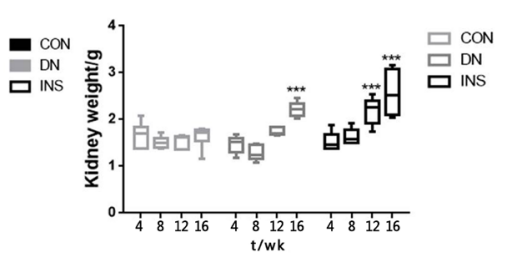

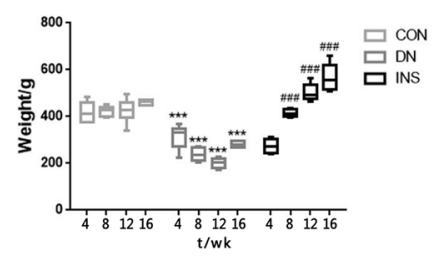

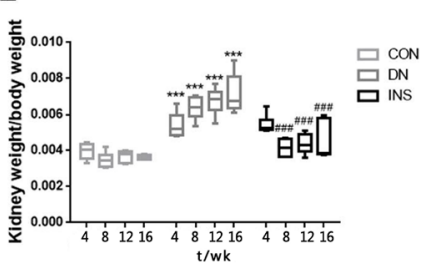

Figure 1. The changes of blood glucose, urinary albumin excretion rate and kidney weight ratio in different stages. (A) Blood glucose level; (B) Urinary albumin excretion rate; (C) Kidney weight; (D) Body weight; (E) Kidney body weight ratio. Abbreviations: CON, normal control group rats; DN, diabetic nephropathy group rats; INS, Insulin treatment group rats. "represents DN or INS VS CON, "represents CON or INS VS DN; ${ }^{*} P<0.05,{ }^{\sharp} P<0.05$.

blood glucose levels. Significantly, diabetic rats showed a steady rise in random blood glucose levels to week 16, with significant differences compared with control and insulin-treated groups (Figure 1A). Compared with the control group, the 24-h urinary albumin excretion rate in the T2DN group increased significantly during the same period, but decreased significantly when insulin was given (Figure 1B). Compared with the control group, renal weight gradually increased in the DN group and insulin groups, which was statistically significant at week 16 , (Df $=54$, $F=7.305, P<0.05$, ANOVA) (Figure 1C). The body weight of DN rats decreased significantly, but kidney weight ratio significantly increased in different stages that compared with the control group (Figure 1D,E).

\subsection{Histopathological changes of renal tissues in diabet- ic rats at different stages}

HE staining found that the changes of softening foci were observed in the hypothalamus of the DN rats, it could be due to diabetes leading to incomplete circulation (Figure 2A). Histopathological examination of kidney from diabetic rats revealed that the mesangial cells and stroma of the glomerulus were increased, and the peripheral renal tubules were damaged to different degrees. Significantly, as the age of the rats, the lesions were being increasingly identified, and glomerulosclerosis with tubular necrosis or atrophy is observed at week 16. Conversely, renal tissue morphology was normal, and the tubulosphere structure was intact in the control group. Treatment with insulin significantly prevented deterioration in the kidney of diabetic rats (Figure 2B).

\subsection{Changes of the expression of POMC in the brain and kidney of DN rats at different stages}

To explore the changes of hypothalamus energy metabolism-related genes of diabetic rats at different stages, immunohistochemical staining was conducted for POMC in brain and kidney sections. With the progression of diabetes, POMC of the appetite-suppressing positive neuron decreased gradually from the 8th week in diabetic rats brains, while it was significantly lower than that of the control group and insulin group at week 16, (Df $=36, F=34.60, P<0.05$, ANOVA) (Figure 3A,B). The renal results showed that the expression of POMC protein levels decreased significantly at different stages of disease in the DN group; POMC increased significantly in the insulin intervention group from week 12, with the insulin having a better effect (Figure 3C,D).

\subsection{Changes of the expression of AgRP in the brain and kidney of DN rats at different stages}

Next, we investigated the protein expression levels of AGRP in brain and kidney tissues of diabetic rats. The results showed that the expression in the hypothalamus region, a positive neuron that promotes appetite, maintained high expression in the DN group at different stages, but insulin decreased its expression, and at week 16 , insulin intervention was significant (Figure 4A,B). Compared with the control and insulin groups, the level of AGRP elevated in DN rats from week 4 to week 8 and then gradually decreased. Insulin significantly reduces AGRP expression in different stages of the disease, and there was a significant difference between week 8 and week 12 (Figure 4C,D).

\section{Discussion}

The present study adds to the existing knowledge on neuroendocrine control from the brain to kidney tissue in DN rates. We focused on the neurogenic-related peptides POMC/ $\mathrm{AgRP}$ in the kidney and found that the expression of POMC in the renal tissues of DN rats was decreased, and AgRP was significantly increased compared with the control group. With the progression of DN, the POMC expression level in brain and renal tissues were gradually decreased, with the 
A

\section{Brain CON}

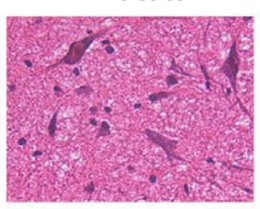

Brain DN

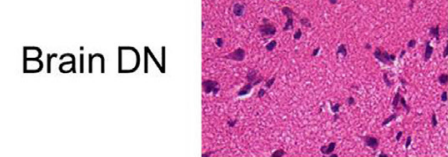

Brain INS

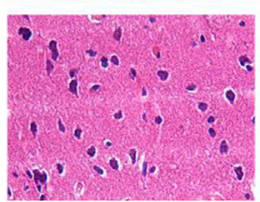

B

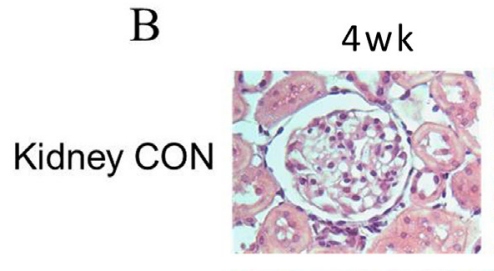

Kidney DN
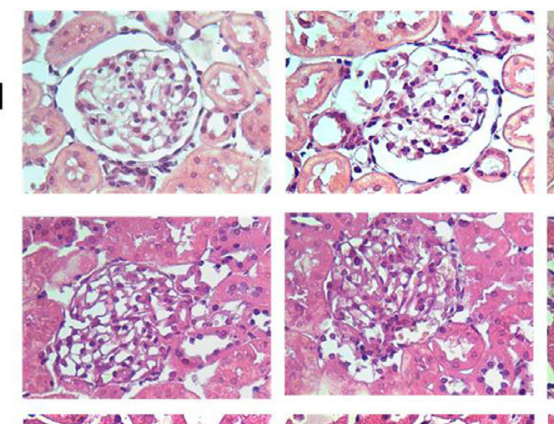

$8 w k$

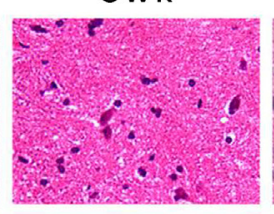

$12 \mathrm{wk}$
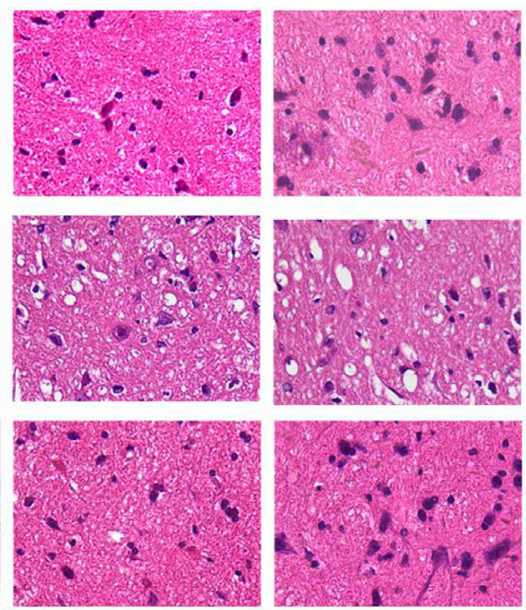

$12 w k$
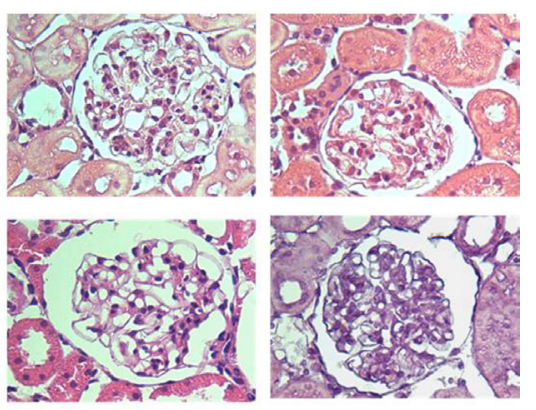

Kidney INS
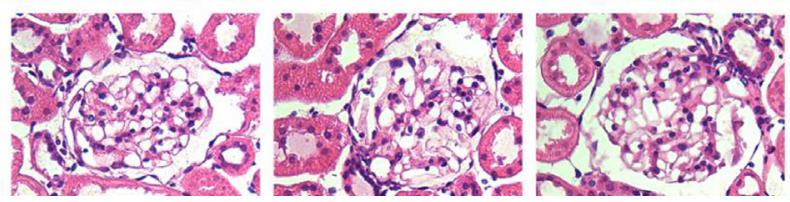

$16 \mathrm{wk}$
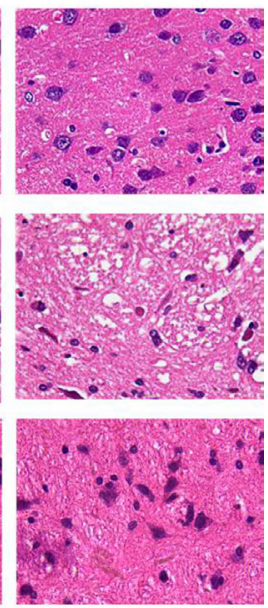

$16 \mathrm{wk}$
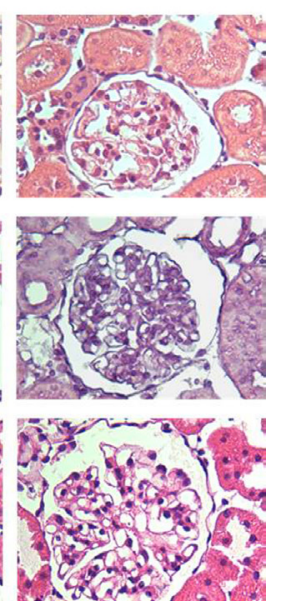

Figure 2. Pathological changes of brain and kidney. (A) Representative brain histology of H\&E staining in brain sections in each group. (magnification 400x); Scale bars, $20 \mu \mathrm{m}$. (B)Representative kidney histology of H\&E staining in kidney sections in each group. (magnification 400x); Scale bars, $20 \mu \mathrm{m}$. CON, normal control group rats; DN, diabetic nephropathy group rats; INS, Insulin treatment group rats.

lowest expression at week 16. No fluctuation was found in brain AgRP expression with the age of DKD rats increasing, but its expression in kidney was highest at week 8 and then decreased gradually. The effect of insulin on normalizing POMC and AgRP expression in brain and renal tissues were also observed in DKD rats. Taken altogether, these data indicate that the abnormal expression of POMC and AgRP in the kidney of DKD rats could be one of the potential factors for kidney injury, leading to DN progression. Further experiments in detail are going on in our laboratory to demonstrate the correlation between POMC and AgRP and DN.

POMC was identified from the cloning of the bovine cDNA by Nakanishi and colleagues in 1978 [13]. POMC can produce at least eight different peptides whose biologic roles are not fully described through enzyme-mediated cleavage that varies among cell types, including $\beta$-endorphin ( $\beta$-EP), melanocyte stimulating hormone (MSH), and adrenocorticotropin (ACTH) [14]. First, hypothalamus and pituitary gland were considered as the production and expression location of POMC. Immunological and mRNA expression studies have found that POMC can also be produced in non-pituitary tissues, such as immune cell and osteoarticular system [1517]. So far, no studies have reported whether POMC can be expressed in the kidney. In our study, it was confirmed for the first time that POMC was expressed consistently in the kidney of rats in control group, but the expression of POMC gradually increased with the age of rats increasing in the brain tissue. Compared with the control group, the expression of POMC in brain and kidney of DKD rats was gradually decreased and of which can be normalized by insulin. Although the biological activity of the skin derived from the blood-secreting pituitary gland was discovered 80 years ago and the neuropeptides isolated 20-40 years ago, our understanding of POMC-derived peptides in non-neuron cell is still rapidly evolving.

As we know, POMC expressed in the brain, especially in the hypothalamus, is an anorexic neuropeptide, which is induced 


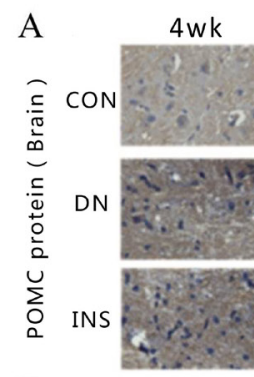

$$
\text { C }
$$

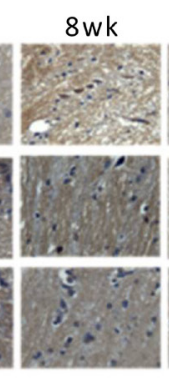

$4 \mathrm{wk}$

$8 w k$
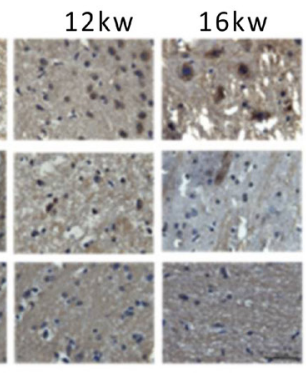

$12 \mathrm{kw} \quad 16 \mathrm{kw}$

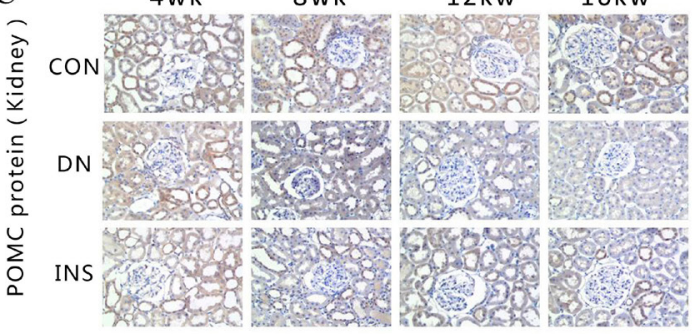

B

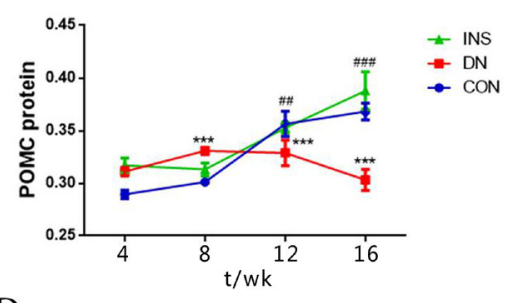

$\mathrm{D}$

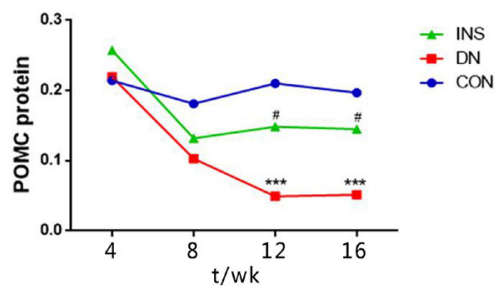

Figure 3. POMC protein expression levels in DN rat kidneys and brains. (A) Expression of POMC in rats' brain by immunohistochemistry (magnification 400x), Data analysis of (B) POMC protein, (C) Expression of POMC in rat kidneys by immunohistochemistry (magnification 400×), Data analysis of (D) POMC protein. CON, normal control group rats; DN, diabetic nephropathy; INS, Insulin treatment group rats; POMC, proopiomelanocortin "represents DN or INS VS CON, " $\# P<0.05$.

A

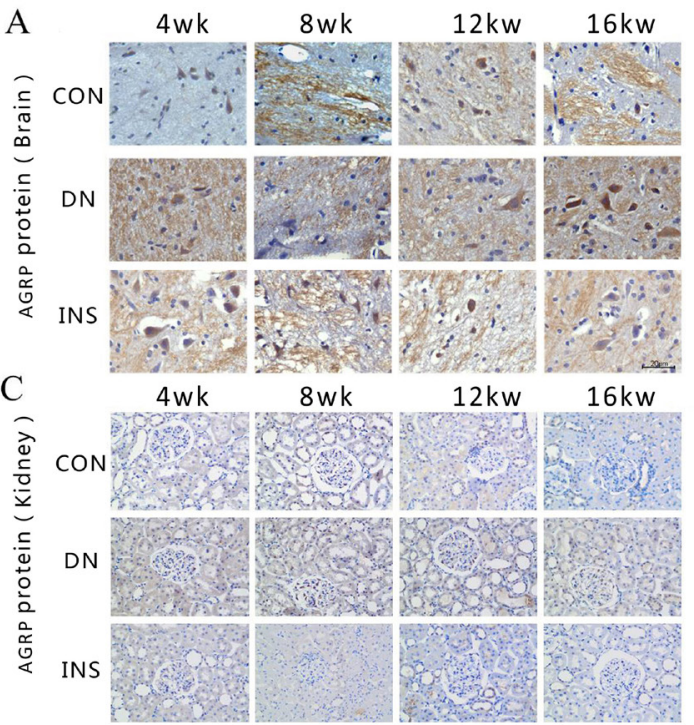

B

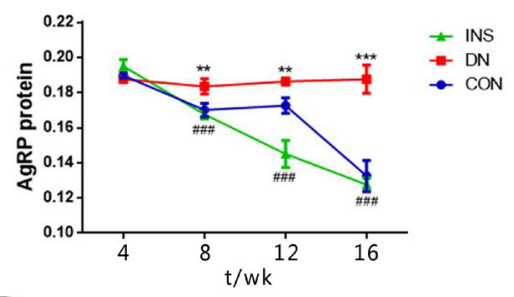

$\mathrm{D}$

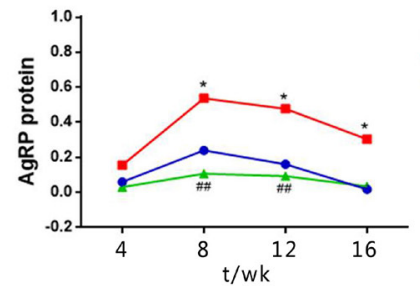

Figure 4. AgRP protein expression levels in DN rat kidneys and brains. (A) Expression of AgRP in rats brain by immunohistochemistry (magnification 400x), Data analysis of (B) AgRP protein, (C) Expression of AgRP in rat kidneys by immunohistochemistry (magnification 400x), Data analysis of (D) AgRP protein. AgRP, agouti-related peptide; CON, normal control group rats; DN, diabetic nephropathy; INS, Insulin treatment group rats. " $r e p r e s e n t s ~ D N$ or INS VS CON, " represents CON or INS VS DN; *P<0.05; ${ }^{*} P<0.05$.

by high fat and high sugar to reduce its expression and promote the increase of food intake [11]. This is consistent with the clinical symptoms of diabetes. From the immunohistochemical results, it is not difficult to find that POMC in DKD rats is mainly expressed in renal tubular epithelial cells, with a small amount expressed in glomerular mesangial cells. These results suggest that $\mathrm{POMC}$ is mainly involved in renal tubular injury in DKD rats. The regulation and processing of POMC in immune cells, as well as the interaction between POMC product peptide and other cytokines, have been well understood [16]. For example, Gądek-Michalska et al. described that IL-1 causes POMC production by immune cells, and the $\mathrm{POMC}$ product, alpha-MSH, in turn, acts functionally as an IL-1 antagonist in immune cells [18]. Most studies on POMC peptide and its receptors in bone focus on the role of melanocorticoid peptide, rather than the POMC expression [19]. The immunoreactivity of POMC in chondrocytes and synovium cells of the knee joint of rats treated with POMC 
adenovirus vector was significantly enhanced, suggesting that POMC plays an anti-inflammatory role, but the specific mechanism of kidney POMC expression remains to be further studied.

AgRP, an orexigenic neuropeptide, acts as an antagonist in the central melanocortin system and binds to melanocortin receptor 4 (MC4R), antagonizing a-MSH-mediated $\mathrm{G}$ protein activation, inhibiting anorexia in animals, and weight regulation[20]. In addition, AgRP can selectively increase the consumption of high-fat foods [21]. Chantal Charbonneau et al. reported that feeding upregulated the expression of AgRP in epididymal fat [22]. Current studies have not reported the AgRP expression in the kidney. However, we explored the increased the expression of AgRP protein both in brain and kidney tissue of DKD rats. Similarly, AgRP is mostly expressed in renal tubular epithelial cells, less in glomerular cells, but the latter more than POMC expression. Of note, $\mathrm{Qu}$ et al. found that the expression of MC4R is higher in the brain than in the kidneys, and its expression in the liver is lowest [23]. In this study, it was found that the expression of POMC in the brain and kidney of T2DN group decreased continuously, while the expression of AGRP was higher than that of control and insulin-treated groups. In addition, the present study also found an increase in renal/body weight in DKD rats compared with the control group and the insulin group. It remains to be further investigated whether this is related to the role of renal AgRP combined with MC4R.

\section{Conclusion}

There are a few reports in the literature about the expression of POMC/AgRP in kidney tissue both in normal rats or DKD rats. Our study showed different expression levels of two neuropeptides at different stages of DKD progression, and their expressions normalized significantly in the insulin group. This revealed that insulin may prevent the progression of kidney injury by inhibiting the expression of neuropeptides expressed in situ in the kidney. This study is one of a series from our group to determine the neuroendocrine regulation of "brain-kidney" axis on the pathogenesis of DN. However, there are also some issues that should be further discussed. For example, the types of cells in which POMC was expressed in the kidney were expressed in nerve fibers or other kidney cells. Also, other methods such as RT-PCR will also be carried out to demonstrate the results of this study. In total, further research studies to explore a new strategy for the treatment of DN are underway in our laboratory.

\section{Acknowledgments}

National Natural Science Foundation of China (81760134, 81960139), Guizhou Provincial Science and Technology Committee (QKHPTRC2018-5636, QKHZC2019-2801, QKHTF[2020]1Y306, QKHTF(2020)1Y304). Thanks to Prof. Xiaobing Gao (Department of Comparative Medicine, Yale University, USA) for the revision of this paper.

\section{Author Contributions}

Shasha Liu, and Yan Zha conceived and designed the experiments; Jingjing Da, Jiayu Li and Rong Dong performed the experiments; Shasha Liu and Jingjing Da analyzed the data and wrote the paper; Yan Zha revised article.

Conflicts of Interest

Yan Zha is an Editorial Board Member of the journal. The authors declare no other conflict of interest.

\section{REFERENCES}

[1] Tziomalos K, Athyros VG. Diabetic nephropathy: New risk factors and improvements in diagnosis. Rev Diabet Stud 2015; 12:110-8.

[2] Saeedi P, Petersohn I, Salpea P, Malanda B, Karuranga S, Unwin $\mathrm{N}$, et al. Global and regional diabetes prevalence estimates for 2019 and projections for 2030 and 2045: Results from the International Diabetes Federation Diabetes Atlas, 9th edition. Diabetes Res Clin Pract 2019; 157:107843.

[3] Williams R, Karuranga S, Malanda B, Saeedi P, Basit A, Besançon $\mathrm{S}$, et al. Global and regional estimates and projections of diabetesrelated health expenditure: Results from the International Diabetes Federation Diabetes Atlas, 9th edition. Diabetes Res Clin Pract 2020; 162:108072
[4] Dekkers CCJ, Gansevoort RT, Heerspink HJL. New diabetes therapies and diabetic kidney disease progression: The role of SGLT2 inhibitors. Curr Diab Rep 2018; 18:27.

[5] Alvarez-Bolado G. Development of neuroendocrine neurons in the mammalian hypothalamus. Cell Tissue Res 2019; 375:23-39.

[6] Morita-Takemura S, Wanaka A. Blood-to-brain communication in the hypothalamus for energy intake regulation. Neurochem Int 2019; 128:135-42.

[7] Timper K, Brüning JC. Hypothalamic circuits regulating appetite and energy homeostasis: Pathways to obesity. Dis Model Mech 2017; 10:679-89.

[8] Kaneko K, Soty M, Zitoun C, Duchampt A, Silva M, Philippe E, et al. The role of kidney in the inter-organ coordination of endogenous 
glucose production during fasting. Mol Metab 2018; 16:203-12.

[9] van Schaftingen E, Gerin I. The glucose-6-phosphatase system. Biochem J 2002; 362:513-32.

[10] Clar J, Gri B, Calderaro J, Birling MC, Hérault Y, Smit GP, et al. Targeted deletion of kidney glucose- 6 phosphatase leads to nephropathy. Kidney Int 2014; 86:747-56.

[11] Zhan C. POMC neurons: Feeding, energy metabolism, and beyond. Adv Exp Med Biol 2018; 1090:17-29.

[12] Clark AJ. 60 YEARS OF POMC: The proopiomelanocortin gene: discovery, deletion and disease. J Mol Endocrinol 2016; 56:T27-37. [13] Balthasar N, Dalgaard LT, Lee CE, Yu J, Funahashi H, Williams T, et al. Divergence of melanocortin pathways in the control of food intake and energy expenditure. Cell 2005; 123:493-505.

[14] Raffin-Sanson ML, de Keyzer $Y$, Bertagna $X$ Proopiomelanocortin, a polypeptide precursor with multiple functions: From physiology to pathological conditions. Eur J Endocrinol 2003; 149:79-90.

[15] Wintzen M, Gilchrest BA. Proopiomelanocortin, its derived peptides, and the skin. J Invest Dermatol 1996; 106:3-10.

[16] Wang W, Guo DY, Lin YJ, Tao YX. Melanocortin regulation of inflammation. Front Endocrinol (Lausanne) 2019; 10:683.
[17] Blalock JE. Proopiomelanocortin and the immune-neuroendocrine connection. Ann N Y Acad Sci 1999; 885:161-72.

[18] Gądek-Michalska A, Bugajski J. Interleukin-1 (IL-1) in stress-induced activation of limbic-hypothalamic-pituitary adrenal axis. Pharmacol Rep 2010; 62:969-82.

[19] Grässel S, Muschter D. Do neuroendocrine peptides and their receptors qualify as novel therapeutic targets in osteoarthritis? Int J Mol Sci 2018; 19:367.

[20] Su Z, Alhadeff AL, Betley JN. Nutritive, post-ingestive signals are the primary regulators of AgRP neuron activity. Cell Rep 2017; 21:2724-36.

[21] Shibata M, Banno R, Sugiyama M, Tominaga T, Onoue T, Tsunekawa $\mathrm{T}$, et al. AgRP neuron-specific deletion of glucocorticoid receptor leads to increased energy expenditure and decreased body weight in female mice on a high-fat diet. Endocrinology 2016; 157:1457-66.

[22] Charbonneau C, Bai F, Richards BS, Argyropoulos G. Central and peripheral interactions between the agouti-related protein and leptin. Biochem Biophys Res Commun 2004; 319:518-24.

[23] Qu H, Li J, Chen W, Li Y, Jiang Q, Jiang H, et al. Differential expression of the melanocortin-4 receptor in male and female C57BL/6J mice. Mol Biol Rep 2014; 41:3245-56. 